\title{
Avances y retrocesos en la inclusión laboral de personas con discapacidad en Argentina entre 2011 y 2019
}

\author{
Advances and setbacks in the labor inclusion of people with disabilities \\ in Argentina between 2011 and 2019
}

\begin{abstract}
Resumen
El artículo propone analizar el surgimiento del sistema de cuotas en el empleo público en Argentina e indagar en la eficacia de esta política pública entre 2011 -cuando comienza el segundo gobierno de Cristina Fernández de Kirchner- y 2019, cuando concluye la gestión de Mauricio Macri. Nos interesa describir y evidenciar los datos disponibles de las vacantes ocupadas y compartir interrogantes en torno al cumplimiento de la política, focalizando en su eficacia. Inicialmente, en el proceso de historización se identifican los actores sociales que han sido fundamentales en el proceso de inscripción en la agenda estatal de los derechos de las personas con discapacidad. Posteriormente, se analiza desde la categoría de capacidad estatal los grados de cumplimiento de lo establecido en la normativa específica, a partir de los datos proporcionados por la Oficina Nacional de Empleo Público de Argentina. Por último, se comparten reflexiones en torno a cómo esto se entrelaza a las formas en que social y culturalmente se valora el trabajo, los cuerpos de los sujetos y las expectativas de funcionamiento corporal.
\end{abstract}

\section{Palabras clave}

Inclusión laboral, discapacidad, Argentina, capacidad estatal, cumplimiento sistema de cuotas.

\begin{abstract}
The article proposes to analyze the emergence of the quota system in public employment in Argentina and investigate the effectiveness of this public policy between 2011 -when the second government of Cristina Fernández de Kirchner begins- and 2019, when Mauricio Macri's administration ends. We are interested in describing and highlighting the data available on the vacancies filled and sharing questions about compliance with the policy, focusing on its effectiveness. Initially, in the historicization process, the social actors that have been fundamental in the process of registration in the state agenda of the rights of persons with disabilities are identified. Subsequently, the degree of compliance with the provisions of the specific regulations is analyzed from the category of state capacity, based on the data provided by the National Office of Public Employment of Argentina. Finally, reflections are shared about how this is intertwined with the ways in which work, the bodies of the subjects and the expectations of bodily functioning are socially and culturally valued.
\end{abstract}

\section{Keywords}

Labor inclusion, disability, Argentina, state capacity, compliance with the quota system.

\author{
Paula Mara Danel \\ $<$ danelpaula@hotmail.com>
}

Consejo Nacional de Investigaciones Científicas y Técnicas (CONICET). Argentina

\section{Sergio Hernán Blogna Tistuzza \\ <sergio_blogna@hotmail.com> \\ Universidad Nacional de Quilmes. Argentina}

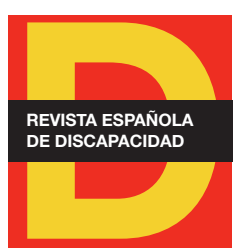

Para citar:

Danel, P. M. y Blogna, S. H. (2021). Avances y retrocesos en la inclusión laboral de personas con discapacidad en Argentina entre 2011 y 2019. Revista Española de Discapacidad, 9(2), pp. 45-63.

Doi: <https://doi.org/10.5569/23405104.09.02.03>

Fecha de recepción: 09-01-2021 Fecha de aceptación: 01-10-2021 


\section{Introducción}

“La perspectiva 'capacitista' define la discapacidad en términos de deficiencia y desvío de la norma humana" (Ferrier y Muller, 2008). Según Soledad Arnau, retomando a G. Wolbring, estos posicionamientos capacitistas se centran en una "plus valoración" de las capacidades humanas, siendo que a más capacidades más valorado el individuo será, quedando por fuera de los circuitos de reproducción social aquellos que por sus capacidades devaluadas sufren una "infravalorización”. Esta idea de cuerpo minusválido, discapacitado, deficitario, aparece como parte del proceso de distribución y división de la fuerza de trabajo, en donde el capitalismo moderno segrega a los cuerpos incapaces y no productivos, destinándolos (desde la noción médica-rehabilitadora) a la institucionalización y al apartamiento de todo tipo de participación socio política, cultural y económica (Arnau, 2013). Al cuerpo con discapacidad se opone "otro" cuerpo no-discapacitado, que aparece como normal, completo, ideal, autónomo e independiente y, sobre todo, productivo en el proceso de trabajo. Estos conceptos nos permiten comprender por qué las personas con discapacidad han sido históricamente excluidas, en tanto han sido vistas como divergentes, desiguales, sufrientes, deficitarias, oprimidas (Calfunao et al., 2019). En simples palabras, "el capacitismo coloca a las personas en situación de discapacidad en exclusivos itinerarios terapéuticos, y los excluye del trabajo asalariado" (Danel, 2019, p. 76).

Nuestro modo de comprensión de la discapacidad se inscribe en perspectivas críticas de las ciencias sociales que colocan a las relaciones sociales, a las condiciones materiales de vida, como productoras de la discapacidad. Brogna (2006) nos invita a pensar cómo se produce la existencia de la discapacidad, y nos advierte que la misma toma cuerpo siempre de manera situada. Los consensos internacionales proponen la idea de persona con discapacidad, la que se respalda en la Convención sobre los derechos de las personas con discapacidad (Organización de las Naciones Unidas, 2006). Si bien tomamos el marco normativo como herramienta sustancial para pensar las políticas y las acciones estatales, creemos que la idea de situación de discapacidad expresa de manera más fiel los procesamientos sociohistóricos e ideológicos presentes en la producción de discapacidad, y al mismo tiempo permite identificar las relaciones sociales, las agencialidades y las constricciones.

En tal sentido, al intentar indagar en esa situación de "exclusión" en el ámbito laboral que atraviesan las personas con discapacidad en Argentina, encontramos que no existen datos oficiales de las últimas décadas que permitan un análisis confiable de la evolución de la tasa de empleo y otros indicadores laborales (Mareño, 2015). Los datos disponibles difieren entre sí, aunque todos coinciden en que el colectivo con discapacidad se enfrenta a un escenario caracterizado por: escasas oportunidades de empleo genuino, desventajas significativas en el mercado laboral, bajos ingresos, asignación de tareas no cualificadas y de escasa productividad, omisión de derechos laborales, subocupación horaria y empleos de baja calidad y altamente inestables (International Disability Rights Monitor, 2004; Samaniego, 2006; O’Reilly, 2007; Bueno y Andreu, 2009; Organización Mundial de la Salud, 2011; REDI et al., 2012 y Blogna Tistuzza, 2020).

Entendemos, junto a Dussel (2000) y a Ocampo (2018) que la exclusión laboral se vincula a procesos previos de inclusión excluyente, en tanto marca de las experiencias educativas de las personas en situación de discapacidad. Eso supone identificar las líneas de continuidad entre las dinámicas de inclusión excluyente escolares, con las que se presentan en la vida adulta asociado al trabajo. 
Según el Informe Mundial sobre la Discapacidad, un estudio del año 2010 de la Organización para la Cooperación y el Desarrollo Económico comprobó que, en 27 países, las personas definidas como discapacitadas en edad de trabajar, en comparación con el resto de la población de la misma edad, tienen peores oportunidades de empleo (Organización Mundial de la Salud, 2011). La tasa de empleo es del 44\% en el primer caso contra el $75 \%$ del resto. Con respecto a la región Iberoamericana, la publicación plantea que "las tasas de actividad y empleo de las personas con discapacidad, aunque difieren de unos países a otros, son en general sensiblemente inferiores a las del resto de la población" (Organización Iberoamericana de Seguridad Social, 2014). En esa línea, limitando el análisis a los países de Latinoamérica, dos estudios que abordan el reconocimiento y ejercicio de derechos fundamentales en este sector de la población, señalan que "uno de los principales problemas que enfrenta la población con discapacidad en la región es el desempleo y la marginación laboral. Según la información obtenida, en promedio, alrededor del $70 \%$ de las personas con discapacidad de la región están desempleadas o excluidas de la fuerza laboral" (International Disability Rights Monitor, 2004; Stang, 2011).

En Argentina, entre los años 2002 y 2003, el Instituto Nacional de Estadística y Censos de la República Argentina (INDEC) Ilevó a cabo la primera Encuesta Nacional de Discapacitados, conocida como ENDI. Este estudio informó una tasa de desocupación de la población con discapacidad del 15,8\% y una tasa de empleo del 24\%. Es decir, la mayor proporción del colectivo con discapacidad -alrededor de un 60\%- no tenía trabajo ni lo buscaba (INDEC, 2005). Y, entre las personas con discapacidad motora mayores de 14 años y ocupadas (el 22,4\%), prácticamente la mitad son obreros o empleados y el resto trabajadores por cuenta propia, pero no se registran patrones (Ferrante, 2007, p. 10).

En segundo lugar, el INDEC llevó a cabo, en 2018, el Estudio Nacional sobre el perfil de las personas con discapacidad. Este informe estadístico revela que la tasa de desocupación es muy similar a la de la población en general: $10,3 \%$ en personas con discapacidad frente al 9,1\%. Sin embargo, el resto de los indicadores sí muestran diferencias relevantes. Por ejemplo, la tasa de empleo de las personas con discapacidad, del $32,2 \%$, es menor a la del resto de la población, 42,2\% (INDEC, 2018a). Sin embargo, los datos desagregados muestran que esa tasa para hombres con discapacidad es del $40,3 \%$ y para mujeres con discapacidad del 25,8\%, lo que revela la discriminación múltiple que sufren en particular las mujeres (Sheppard, 2011). En relación con la tasa de inactividad vemos que, para 2018, la tasa para la población económicamente activa era del 55,9\% (Banco Mundial, 2018), mientras que para personas con discapacidad era del 64,1\% en el país, 55,6\% para hombres y del 70,9\% para mujeres (INDEC, 2018b). Este Estudio Nacional sobre el perfil de las personas con discapacidad ha revelado los motivos de las personas con discapacidad que no buscan trabajo: el $24,2 \%$ posee un ingreso de tipo previsional (jubilación o pensión), incompatible con cualquier otro tipo de ingresos económicos. El 13,6\% considera que no va a conseguir trabajo a causa de la discapacidad y el 9,8\% -en promedio- no inicia una búsqueda laboral porque se dedica al cuidado del hogar (1,1\% son varones y el 15,7\% mujeres) (INDEC, 2018a, p. 132). Estos indicadores coinciden con lo presentado por diversas organizaciones de la sociedad civil ${ }^{1}$ ante el Comité sobre los derechos de las personas con discapacidad de Naciones Unidas: una "gran cantidad de personas con discapacidad en la Argentina están desempleadas y no buscan trabajo ya que saben que es muy difícil encontrarlo y, en consecuencia, no aparecen entre la población económicamente activa" (REDI et al., 2012, p. 69). Dicha población se retira involuntariamente del mercado laboral ante la falta de oportunidades y se constituye como población des-

1. Red por los Derechos de las Personas con Discapacidad, Centro de Estudios Legales y Sociales, Federación Argentina de Instituciones de Ciegos y Amblíopes, Federación Argentina de Entidades Pro Atención a las Personas con Discapacidad Intelectual y Asociación por los Derechos Civiles. 
ocupada oculta en la inactividad. El mencionado informe agrega además que una medición correcta para nuestro país que incluya a la totalidad de las personas con discapacidad en edad de trabajar arrojaría una tasa de desocupación del $80 \%$, en coincidencia con las estimaciones de la Organización Internacional del Trabajo (Organización Internacional del Trabajo, 2003).

En tercer lugar, desde el año 2009, el Ministerio de Salud de la Nación comenzó a publicar el Registro Nacional de Personas con Discapacidad, el cual sólo contiene los datos de las personas con discapacidad que tramitan y efectivamente obtienen el certificado de discapacidad. Esta publicación contiene mucha información sobre diferentes aspectos socioeconómicos del colectivo en cuestión, pero al incluir sólo a las personas con discapacidad certificada se limita a un universo muy pequeño en proporción al total (181.969 personas para el año 2014). En particular, el anuario estadístico muestra que el $71 \%$ de la población económicamente activa tiene empleo, pero ese número se reduce al $12 \%$ en el colectivo con discapacidad mayor de 14 años. Esta cifra incluso disminuye al 10,99\% en las mujeres. Estos datos muestran indicadores similares a los previamente relevados por el INDEC, en los diferentes informes señalados.

En síntesis, como confirman las diferentes fuentes, las personas cuyos cuerpos son "infravalorados" por sus capacidades devaluadas sufren el desempleo en una proporción mucho mayor frente a los "otros" cuerpos no-discapacitados. Siguiendo los aportes de Platero (2012), señalamos que las políticas de acceso al trabajo resultan desde una perspectiva monofocal. En consecuencia, "las políticas que tratan de compensar discriminaciones históricas y estructurales están reproduciendo la desigualdad, al ignorar las jerarquías de poder que naturalizan y así produciendo unos efectos no deseados que se traducen en exclusiones concretas" (Platero, 2012, p. 45).

El objetivo del presente trabajo es analizar el surgimiento del sistema de cuotas en el empleo público en Argentina, a nivel nacional. Asimismo, nos proponemos indagar en el cumplimiento de esta política pública entre 2011 -cuando comienza el segundo gobierno de Cristina Fernández de Kirchner-y 2019, cuando concluye la gestión de Mauricio Macri. Nos interesa describir y evidenciar los datos disponibles de las vacantes ocupadas y compartir interrogantes en torno al cumplimiento de la política, focalizando en su eficacia. En ese marco, destacamos junto a Sconfienza (2016) que las políticas de empleo implementadas en nuestro país se concentran en cuatro pilares fundamentales, como la generación de empleos, los subsidios al salario, la capacitación y la intermediación laboral. En este trabajo, nos interesa especialmente observar la generación de empleos destinados a personas en situación de discapacidad, en el marco del sistema de cuotas.

\section{Surgimiento del sistema de cuotas en Argentina}

En 1956 se creó la Comisión Nacional de Rehabilitación del Lisiado (CONAREL), la primera institución oficial dedicada a la rehabilitación de las personas con discapacidad del aparato locomotor, construida bajo el modelo médico hegemónico a nivel local (Álvarez, 2013). A través de esta comisión, se crearon el Instituto de Rehabilitación del Lisiado (IREL), el Centro Nacional de Rehabilitación Respiratoria "María Ferrer", las Escuelas de Terapia Ocupacional y Ortesis y Prótesis, y carreras auxiliares de la fisiatría. "Para ello, se contratarían a profesores del exterior especializados y se becarían a profesionales argentinos para que estudiaran en el exterior" (Ferrante, 2012, p. 42). 
Durante el período 1967-1973 se hicieron públicas las constantes quejas de las personas con discapacidad que reclamaban una gestión real de la CONAREL en favor del derecho al trabajo: "[...] el 90 por ciento de los lisiados rehabilitados no trabaja. Gran cantidad piden limosna y otros tantos viven del mantenimiento de la familia. Muchos quedan en cotolengos, asilos. Es el lisiado un vago? [...] Ya es hora de que nos rebelemos. De que se dé a conocer nuestra fuerza y nuestra razón. [...] Ya estamos cansados de promesas. El hambre y la necesidad nos esperan. Nosotros también somos ciudadanos" (Club Marcelo J. Fitte, 1967). En concreto, exigían que el Estado instrumentara un cupo laboral de personas con discapacidad, inspirado en la ley británica homóloga. Sin embargo, las autoridades de la CONAREL no atendieron el reclamo (Ferrante, 2012, p. 45).

En este contexto, los lisiados ${ }^{2}$ que trabajaban en el Hogar Escuela de la Asociación Cooperadora del Instituto de Rehabilitación (ACIR), de clase baja y de provincias del interior del país, cuestionaron la explotación a la que eran sometidos por parte de la escuela de oficios que les pagaban un pecunio que sólo les alcanzaba para cigarrillos. También denunciaron que no les daban de comer y que sólo realizaban beneficencia a través de ellos (Sbriller, 2016). Exigían romper con el modelo de dominación ejercido por el modelo médico hegemónico y caritativo sobre la discapacidad, aquel que concilió la idea de la concepción capitalista del hombre sano y productivo para ser reconocido como trabajador, su lema: "los lisiados seremos artífices de nuestro propio destino, nunca un instrumento de la ambición de nadie". En 1971, fundaron su propio espacio, el Club Integración Deportista Discapacitados de Argentina (CIDEDA). Esta organización nucleaba a mendigos, trabajadores ambulantes que tomaban alcohol, "eran los negros del bajo" (Sbriller, 2016, p. 101). CIDEDA representó la lucha de clases y constituyó el Frente Rengo Peronista integrado, entre otros, por José Poblete Roa, Gilberto Rengel Ponce y Alejandro Alonso, que ante el pedido de Juan Domingo Perón pasó a denominarse Frente de Lisiados Peronistas (FLP), una agrupación de personas con y sin discapacidad que, sumándose a reclamos colectivos de la época, reivindicaba los derechos sociales (Bregain, 2012). Como bien describe Martín Caparrós, esta organización llegó a estar constituida por más de 200 personas que concurrían a las marchas con sus muletas, sillas de ruedas y lazarillos (Caparrós, 1999). En 1974, el Frente de Lisiados Peronistas denunció las condiciones laborales de explotación que se sufrían en la escuela profesional que funcionaba en la calle Ramsay. Con este reclamo se buscaba interpelar las formas legítimas de acción colectiva: sus prácticas militantes, las acciones de resistencia y su origen de clase proletaria marcan un itinerario de participación política cuya bandera será el derecho a trabajar (Danel, 2019, p. 86).

Con la muerte del entonces presidente Juan Domingo Perón en julio de 1974 y la persecución a militantes de izquierda por parte de la Alianza Anticomunista Argentina (Joly, 2008, p. 34), el Frente de Lisiados Peronistas se disolvió y se reconvirtió en la Unión Nacional Socioeconómica del Lisiado (UNSEL). La adopción de este nombre expresaba un cuestionamiento a la mirada médica de la discapacidad, ya que desplazaba el "problema de la discapacidad del ámbito prestacional y médico e intervenía en las relaciones de fuerza que definían cuál era el cuerpo legítimo, rompiendo con el esquema dualista en el cual descansa la ideología de la normalidad [...]" (Ferrante, 2012, p. 47). Esta nueva organización nucleó a las organizaciones de las más diversas discapacidades con el propósito de accionar políticamente (Sbriller, 2016) y logró que el Honorable Senado de la Nación aprobara, el 12 de septiembre de 1974, un proyecto de ley que contemplara "la necesidad de una solidaridad nacional que obligue al Estado a favorecer la integración del discapacitado en la vida profesional". Durante el debate, el senador Oraldo Britos destacó el rol "de los discapacitados en la elaboración de la ley, lo que coincide con el deseo de los interesados" (Bregain, 2012). Y sostuvo que "este proyecto de ley es, simplemente, el fruto del esfuerzo de los compañeros discapacitados, nucleados en la

2. La referencia como lisiados responde al modo en que hacia mediados del siglo XX se denominaba a las personas en situación de discapacidad. Se conserva la nominación a fin de orientar al lector en torno al momento socio histórico de referencia. 
organización denominada UNSEL" (Honorable Senado de la Nación Argentina, 1974, p. 1846). Unos días más tarde, el 30 de septiembre, la Honorable Cámara de Diputados de la Nación consideró esta iniciativa y la convirtió en ley sin ningún debate (Honorable Cámara de Diputados de la Nación, 1974, pp. 3552-3554). De esta manera, vio la luz la primera ley laboral para personas con discapacidad, la Ley N ${ }^{\circ} 20.923$ (Rengel, 2011). Esta norma creó, con carácter de entidad descentralizada dependiente del Ministerio de Trabajo, la Comisión Nacional del Discapacitado, integrada por dos representantes de centrales de trabajadores, dos representantes del Gobierno nacional y cuatro representantes de "entidades de discapacitados" (Bregain, 2010). Asimismo, estableció que "todo organismo o repartición de la administración nacional y toda empresa estatal, mixta, privada quedan obligados a ocupar mano de obra hasta un cuatro por ciento (4\%) como mínimo del total de plazas de trabajo existentes, proporcionando empleo a discapacitados". Este artículo "cambiaba la historia" y fue celebrado por el colectivo que la impulsó (Alonso y Cuenya, 1974, p. 35).

A principios de 1975 se organizó una comisión para preparar la reglamentación de la Ley № 20.923, integrada por los miembros de la UNSEL. Sin embargo, la llegada de la dictadura cívico militar, el 24 de marzo de 1976, dejó truncado este proceso y se comenzó a perseguir a inválidos y lisiados ${ }^{3}$ (Comisión Nacional sobre la Desaparición de Personas, 2010). "La dictadura no sólo eliminó físicamente a los 'Rengos de Perón' sino también aquellas reivindicaciones políticas que el colectivo más amplio de la USEL había logrado. La reivindicación de la USEL era de avanzada porque reconocían el carácter político de la discapacidad" (Ferrante, 2012, p. 48).

Durante este período de gobierno dictatorial (1976-1983), Argentina fue designada como el miembro del Comité de las Naciones Unidas encargado de la preparación y organización del Año Internacional de los Impedidos (1981), el cual solicitaba a los distintos países el estudio de las posibilidades de adopción de normas acordes con los principios y recomendaciones emanadas de los entes internacionales. Aquel impulso internacional fue acompañado por organizaciones como la Federación Argentina de Entidades Pro-Atención a las Personas con Discapacidad Intelectual (FENDIM) y encontró particular receptividad en algunos altos oficiales de las Fuerzas Armadas. Se creó la Corporación Argentina de Discapacitados, bajo la tutela de la directora nacional de rehabilitación, la cual redactó un anteproyecto de ley integral de la temática de la discapacidad ${ }^{4}$ (Fundación Par, 2008, pág. 87; Acuña y Bulit, 2010).

Finalmente, en 1981, el régimen militar dictó el Decreto-Ley № 22.431, constituyendo el "sistema de protección de los discapacitados", y lo hizo para mostrar en el plano internacional que en el país se respetaban los derechos humanos (Bregain, 2010). Esta norma cristalizó la mirada médica, naturalizando los requisitos de entrada al campo: poseer una discapacidad estatalmente certificada, poseer un apto psico-físico y no ser "peligroso para terceros" (Ferrante, 2012). El Estado, como detentador de la violencia simbólica (Bourdieu, 1999), definió la discapacidad como un déficit anclado en un organismo individual, médicamente certificado, que genera una alteración funcional temporaria y permanente que significa una "situación de desventaja en función de la edad y el medio social” (Ley № 22.431, artículo 2). Su pilar es un paradigma normalizador, entendido como la posibilidad de que las personas "discapacitadas" lleven una existencia tan próxima a lo normal como sea posible (Rubio, 2009). También es importante destacar que esta norma habilitaba el acceso a los "servicios" sólo a aquellos "discapacitados" que poseían un certificado, expedido por una autoridad sanitaria. Esta certificación,

\footnotetext{
3. La referencia como inválidos y lisiados se mantiene como modo de interpelación al lector en torno a la crudeza con la que la dictadura cívico, militar, empresarial y eclesiástica en Argentina persiguió, torturó, desapareció forzadamente y asesinó a los colectivos organizados y militantes. 4. Por entonces, la dictadura militar (1976-1983) había decidido clausurar el Congreso y sustituirlo por una Comisión de Asesoramiento Legislativo (CAL), con funciones consultivas. De acuerdo al art. 5 del Estatuto para el Proceso de Reorganización Nacional se atribuía al Poder Ejecutivo la facultad de sancionar y promulgar leyes.
} 
conocida desde 2001 como Certificado Único de Discapacidad", no precisa "el grado por incapacidad", sino que acredita que la persona tiene una discapacidad y el Estado Nacional debe velar por los beneficios que él mismo le concede (Nassif y Nassif, 2019, p. 65). Con la dictadura cívico-militar se retornó al concepto de discapacidad como secuela a rehabilitar, desconociendo el carácter político marcado por la UNSEL.

\section{Características del sistema de cuotas}

Bertranou (2013; 2015) nos invita a pensar en las capacidades estatales, en tanto aptitud de los entes estatales para alcanzar los fines que le fueron asignados, en el marco de relaciones de poder y que comprenden un conjunto de factores que el autor denomina componentes de capacidad estatal. Traemos esta idea, que entendemos enhebra de manera oportuna la historización del desarrollo de las capacidades estatales en torno a la producción de política de cupo laboral o cuotas en Argentina.

Según la Organización Internacional del Trabajo, el sistema de cupos laborales "parece ser el que más fomenta el empleo de personas con discapacidades" (Organización Internacional del Trabajo, 2014, p. 62). Se pueden implementar de tres maneras diferentes: a) cuota obligatoria acompañada de sanciones efectivas y/o un mecanismo de exigibilidad, como existe en Alemania, Francia, España, Austria, Polonia, en China y en Japón; b) cuota obligatoria sin sanción efectiva, que se aplica en países como Reino Unido y Tailandia; y c) cuota no vinculante basada en una recomendación, como por ejemplo una circular gubernamental. Este sistema se aplicó en los Países Bajos a mediados de la década de 1980 (Organización Internacional del Trabajo, 2014). Tanto Argentina como Bolivia, Brasil, Costa Rica, Ecuador, El Salvador, España, Honduras, Panamá, Paraguay, Perú, Portugal, Uruguay y Venezuela cuentan con este sistema de cuotas que van del $2 \%$ al $7 \%$ del total de empleo público, y en muchos de estos casos la cuota se extiende también al sector privado (Organización Iberoamericana de Seguridad Social, 2014). En Argentina, el cupo laboral para personas con discapacidad sólo está regulado para el ámbito público, es de cumplimiento obligatorio y posee sanciones efectivas y/o un mecanismo de exigibilidad. En el presente trabajo nos interrogamos sobre la eficacia del cumplimiento de las cuotas e invitamos para futuros estudios, a profundizar en los mecanismos desarrollados en torno a las sanciones.

La Ley $N^{\circ} 22.431$ fue modificada en varias ocasiones, pero respecto del cupo laboral es necesario destacar que, en 2002, el Congreso amplió sus alcances a través de la Ley N²5.689. Desde entonces, es obligación de los tres poderes del Estado -incluyendo organismos descentralizados o autárquicos, los entes públicos no estatales, las empresas del Estado y las empresas privadas concesionarias de servicios públicos- "ocupar personas con discapacidad que reúnan condiciones de idoneidad para el cargo en una proporción no inferior al cuatro por ciento (4 \%) de la totalidad de su personal y a establecer reservas de puestos de trabajo a ser exclusivamente ocupados por ellas". Es importante destacar que, de acuerdo con la ley, este cupo es de cumplimiento obligatorio para el "personal de planta efectiva, para los contratados cualquiera sea la modalidad de contratación y para todas aquellas situaciones en que hubiere tercerización de servicios". Y para dar efectivo cumplimiento a este sistema de cuota, la norma también prevé que las vacantes que se produzcan dentro de las distintas modalidades de contratación "deberán prioritariamente reservarse a

5. El Certificado Único de Discapacidad se encuentra regulado específicamente en la Ley № 25.504 del año 2001. 
las personas con discapacidad que acrediten las condiciones para puesto o cargo que deba cubrirse" e informarse obligatoriamente al Ministerio de Trabajo para que actúe como veedor de los concursos (art. $8^{\circ}$ ).

Por último, queda subrayar que la norma prevé que "en caso de que el ente que efectúa una convocatoria para cubrir puestos de trabajo no tenga relevados y actualizados sus datos sobre la cantidad de cargos cubiertos con personas con discapacidad, se considerará que incumplen el $4 \%$ y los postulantes con discapacidad podrán hacer valer de pleno derecho su prioridad de ingreso a igualdad de mérito. Los responsables de los entes en los que se verifique dicha situación se considerará que incurren en incumplimiento de los deberes de funcionario público, correspondiendo idéntica sanción para los funcionarios de los organismos de regulación y contralor de las empresas privadas concesionarias de servicios públicos" (art. $\left.8^{\circ}\right)$.

Podemos identificar con claridad que la capacidad estatal (Skocpol, 1990), en tanto condición de su autonomía, fue entrelazando las apuestas legislativas que el Estado nacional produjo para configurar el sistema de cuotas, a los actores intervinientes y las posibles sanciones que emanen de su incumplimiento.

Esta política pública, definida en 1981 y actualizada en 2002, introdujo un sistema de cuotas con sanciones, expresivo de un diseño afín al modelo rehabilitador. ¿Por qué decimos esto? Porque, ante la situación de alto desempleo del colectivo con discapacidad, ofrece una medida de discriminación positiva sin analizar, evaluar, atender o responder a los motivos por los cuales el mercado laboral -y las barreras arquitectónicas, culturales y comunicacionales- impiden a las personas con discapacidad realizar su trayectoria educativa y laboral en igualdad de condiciones que el resto. Es cierto que la reserva de cargos en los concursos y en toda contratación constituye una solución a la falta de empleo, pero lo hace sólo parcialmente. A través de la ratificación de la Convención sobre los derechos de las personas con discapacidad, Argentina se comprometió a avanzar con medidas que eliminen toda forma de discriminación. Esto posibilitaría a las personas en situación de discapacidad acceder a un trabajo libremente elegido o aceptado en un mercado laboral que sea abierto inclusivo y accesible.

El desarrollo de capacidades estatales opera en la manifestación más cruda del capacitismo, como lo es el desempleo de las personas adultas en situación de discapacidad. En la dimensión de los resultados de la puesta en acto de esas capacidades se omite la observación sobre los procesos de producción de los sujetos, las posiciones que ocupan en la trama social, sus trayectorias, las marcas de la desigualdad y los recursos a los que acceden/no acceden.

\section{4. ¿Cómo funcionó el sistema de cuotas en Argentina?}

Uno de los criterios que se pueden utilizar para evaluar los resultados de las políticas públicas es la eficacia, es decir, evaluar "en qué medida el área de acción está alcanzando los objetivos, sin considerar necesariamente los recursos asignados para ello (Guzmán, 2007, p. 11). En este caso, para poder indagar si el sistema de cuotas logró la inclusión laboral de personas con discapacidad en el empleo público debemos tener presente que en el año 2010 se reglamentó el artículo $8^{\circ}$ de la Ley $N^{\circ} 22.431$ y se estableció un registro que permite evaluar los resultados de su funcionamiento. Desde los años ochenta hasta entonces no existían datos públicos en el país respecto de la presencia de los trabajadores y trabajadoras con discapacidad en el Estado nacional. 
El Decreto N³12/2010 estableció, concretamente, la obligación de cada organismo de informar a la Dirección Nacional de Gestión de Información y Política Salarial de la Secretaría de Empleo Público del ex Ministerio de Modernización de la Nación un conjunto de datos para elaborar un registro e informe semestral. En particular, se estableció la obligación de informar sobre la cantidad de cargos cubiertos con personas con discapacidad respecto de los totales de la planta permanente y transitoria, y la cantidad de personas con discapacidad contratadas bajo cualquier modalidad respecto del total de los contratos existentes.

En concreto, la regulación determinó que, para asegurar la inclusión laboral de personas con discapacidad en las contrataciones de empleo en el ámbito público nacional, cada vez que una de las jurisdicciones, organismos descentralizados y entidades requiere incorporar personal, debe reservar un cupo mínimo del $4 \%$ de los cargos a cubrir para personas con discapacidad, informar inmediatamente al Ministerio de Trabajo y proceder al concurso laboral. El rol de la cartera laboral cumple dos funciones: ofrece trabajadores con discapacidad registrados en su bolsa de empleo para cubrir los puestos y, por otro lado, actúa como veedor del cumplimiento de la normativa, controlando que se respete la cuota reservada para el colectivo con discapacidad.

Los informes que se han elaborado y publicado en base al Decreto $N^{\circ} 312$, desde 2010 hasta 2019, ofrecen una gran cantidad de información sobre la implementación y los resultados de esta política pública. A continuación, indagaremos sobre estos datos para evaluar el funcionamiento del sistema de cupos durante el período 2011-2015, correspondiente al segundo gobierno de Cristina Fernández de Kirchner, y durante los años 2015-2019, correspondientes al primer y único gobierno de Mauricio Macri.

En diciembre de 2011 comenzó el segundo mandato presidencial de Cristina Fernández de Kirchner. Con esta gestión (2011-2015) se dio inició a un tercer gobierno de la misma fuerza política, que comenzó con la presidencia de Néstor Kirchner en 2003 y continuó con Cristina Fernández de Kirchner (2007-2011). Este período se caracterizó por un "giro a la izquierda", que puso al Estado en el centro de la coordinación social y consolidó el "incremento en la democracia socioeconómica y la ampliación de derechos (vía implementación de políticas públicas concretas" (Trujillo, 2017). Durante este período, los informes de la Oficina Nacional de Empleo Público revelan la siguiente información, que sintetizamos en el cuadro 1.

Cuadro 1. Cupo laboral de personas con discapacidad, entre 2011-2015

\begin{tabular}{|l|c|c|c|c|}
\hline Año & Semestre & Total de trabajadores/as & Trabajadores/as con discapacidad & Cupo laboral (\%) \\
\hline 2011 & $2^{\circ}$ & 341.537 & 2.265 & 0,66 \\
\hline \multirow{3}{*}{2012} & $1^{\circ}$ & 326.413 & 2.390 & 0,73 \\
\cline { 2 - 5 } & $2^{\circ}$ & 315.583 & 2.461 & 0,78 \\
\hline \multirow{3}{*}{2013} & $1^{\circ}$ & 321.386 & 2.471 & 0,77 \\
\cline { 2 - 5 } & $2^{\circ}$ & 328.606 & 2.538 & 0,77 \\
\hline \multirow{3}{*}{2014} & $1^{\circ}$ & 328.351 & 2.588 & 0,79 \\
\hline \multirow{3}{*}{2015} & $2^{\circ}$ & 342.314 & 2.800 & 0,82 \\
\cline { 2 - 5 } & $1^{\circ}$ & 355.130 & 3.054 & 0,86 \\
\hline
\end{tabular}

Fuente: elaboración propia en base a los informes semestrales que elabora la Oficina Nacional de Empleo Público'.

6. Los informes semestrales pueden visualizarse en: www.argentina.gob.ar/jefatura/gestion-y-empleo-publico/registropersonascondiscapacidad. 
Como lo revela el cuadro 1, durante el segundo gobierno de Cristina Fernández de Kirchner se produjo un crecimiento del empleo público del 6,5\%, mientras que el universo de trabajadoras y trabajadores con discapacidad empleados aumentó un $41,1 \%$. Este incremento del empleo público de personas con discapacidad permite explicar el incremento del cumplimiento del cupo laboral, que pasó del 0,66\% en 2011 a $0,9 \%$ en 2015. Los datos confirman que se estaba lejos de cumplir con la cuota $4 \%$ y que durante los años 2011 y 2015 se produjeron mejoras respecto de la inclusión laboral del colectivo con discapacidad. Un dato relevante surge del análisis de los movimientos producidos en el empleo público entre los diferentes semestres. Como lo establece el art. $8^{\circ}$ de la Ley $\mathrm{N}^{\circ} 22.431$, cuando cualquier organismo contrate nuevo personal -en cualquier modalidad de contratación- debe reservar un $4 \%$ de esas vacantes para personas en situación de discapacidad. Si durante estos cinco períodos de crecimiento del empleo la reserva de puestos se hubiese respetado deberíamos ver que, al menos, creció un $4 \%$ la proporción de trabajadores con discapacidad en cada etapa ${ }^{7}$.

Cuadro 2. Evolución del empleo público entre semestres de 2011 y 2015
\begin{tabular}{|l|c|c|c|c|}
\hline Semestres & $\begin{array}{c}\text { Variación de cantidad } \\
\text { de trabajadoras/es }\end{array}$ & $\begin{array}{c}\text { Variación de cantidad } \\
\text { de trabajadoras/es con } \\
\text { discapacidad }\end{array}$ & $\begin{array}{c}\text { Proporción personas } \\
\text { con discapacidad / } \\
\text { Variación }\end{array}$ & $\begin{array}{c}\text { Se cumplió la reserva } \\
\text { del } 4 \%\end{array}$ \\
\hline $2^{\circ} 2011-1^{\circ} 2012$ & -15.124 & 125 & No corresponde & No corresponde \\
\hline $1^{\circ}-2^{\circ} 2012$ & -10.830 & 71 & No corresponde & No corresponde \\
\hline $2^{\circ} 2012-1^{\circ} 2013$ & 5.803 & 10 & $0,17 \%$ & No \\
\hline $1^{\circ}-2^{\circ} 2013$ & 7.220 & 67 & $0,93 \%$ & No \\
\hline $2^{\circ} 2013-1^{\circ} 2014$ & -255 & 50 & No corresponde & No corresponde \\
\hline $1^{\circ}-2^{\circ} 2014$ & 13.963 & 212 & $1,52 \%$ & No \\
\hline $2^{\circ} 2014-1^{\circ} 2015$ & 35.475 & 609 & $1,70 \%$ & No \\
\hline $1^{\circ}-2^{\circ} 2015$ & 8.696 & 143 & $1,60 \%$ & No \\
\hline
\end{tabular}

Fuente: elaboración propia en base a los informes semestrales que elabora la Oficina Nacional de Empleo Público.

El cuadro 2 revela que hubo 5 períodos en los que aumentó el empleo (entre el segundo semestre de 2012 y el primer semestre 2013; entre el primer y el segundo semestre de 2013; y desde el primer semestre de 2014 hasta el segundo semestre de 2015). En ninguno de estos períodos se alcanzó la reserva de cargos del $4 \%$. Sin embargo, en todos los períodos, incluyendo cuando disminuyó el total de trabajadoras y trabajadores, hubo incrementos en la dotación de personas con discapacidad, en un total de 1287 personas.

En síntesis, destacamos que durante el período 2011-2015 no se alcanzó el cupo laboral reglamentario ni se respetó la reserva de cargos (del $4 \%$ ) ante nuevas contrataciones. Sin embargo, se registró un aumento de la contratación de personas con discapacidad ascendente, mejorando el cumplimiento del cupo laboral, del $0,66 \%$ al $0,9 \%$.

7. Los datos provistos en los informes semestrales por parte de la Oficina Nacional de Empleo Público refieren a las altas y bajas totales de personal. Las bajas contemplan toda extinción de contrato, ya sea por despido, jubilación, renuncia u otras causas. Por su parte, las altas contemplan toda nueva relación contractual con nuevas personas, incluyendo todas las modalidades de contratación. 
En diciembre de 2015 llegó al gobierno la Alianza Cambiemos, liderada por el Ingeniero Mauricio Macri, lo que implicó la toma del poder institucional de los sectores corporativos y retornó hacia una nueva oleada de neoliberalismo. Ello representó un pasaje de un Estado garantista de derechos a un Estado que se utiliza como plataforma de los negocios del sector empresarial (García y Gradin, 2016). Este giro a la derecha en la orientación del gobierno (Trujillo, 2017; Cannon y Rangel, 2020), reflotó las recetas viejas utilizadas en los años noventa como la individualización y sectorización de los problemas sociales, y por lo tanto la responsabilización de los sujetos por su situación; el disciplinamiento social, laboral y territorial, que contribuyeron al aumento del conflicto social (Calfunao et al., 2019). Las políticas de esta alianza neoliberal, promercado, generaron una inmediata crisis económica y financiera (Hudson, 2018). Ello se tradujo en severas restricciones presupuestarias y un claro cuestionamiento a la idea de sujeto de derechos que se desplegaba en el periodo que le antecedió. En el contexto señalado observamos procesos orientados a la individuación que toman la forma de una responsabilización. "Cada cual es declarado responsable no solo de su propia suerte... Estos mandatos de individuación encarnan a su vez una exigencia generalizada de "activación" de la voluntad individual" (Castel et al., 2013, p. 47).

El gobierno de Mauricio Macri creó el Ministerio de Modernización a través del Decreto 13/2015, que modifica la Ley de Ministerios. Entre sus principales objetivos se destacan el diseño de una carrera profesional para el empleado público basada en el mérito y la capacitación y la gestión de resultados (Blutman y Hoya, 2019).

Conjuntamente, se dictó el llamado Plan de Modernización del Estado mediante el Decreto 434/16, el cual establecía como una de sus prioridades la revalorización y optimización del empleo público, a través de "la profesionalización del empleo público mediante la constante y creciente capacitación del personal, la incorporación de tecnologías y la construcción de una nueva cultura organizacional”. Tengamos presente que el expresidente Macri, en su discurso ante la Asamblea Legislativa, el $1^{\circ}$ de marzo de 2016, afirmó: "encontramos un Estado plagado de clientelismo, de despilfarro y corrupción. Un Estado que se puso al servicio de la militancia política y que destruyó el valor de la carrera pública" (Honorable Senado de la Nación, 2016). Sin embargo, en la práctica este plan se concentró "en políticas de achicamiento de la planta de personal" (Blutman y Hoya, 2019). En los primeros cuatro meses del gobierno de Mauricio Macri, 127.000 trabajadoras y trabajadores perdieron su empleo, de los cuáles un tercio se produjo por despidos en el sector público (Fuertes y Ferreyra, 2016). Respecto del nombramiento de funcionarios políticos en todos los cargos directivos, en vez de llamar a concursos públicos para su selección se profundizó la discrecionalidad (Chudnovsky, 2017). Mientras que en el año 2015 la modalidad de designación de la alta dirección pública de forma transitoria alcanzaba un 94 \%, en 2016 ese número llegó al 97\% y, en 2017, a sólo el $98 \%$ (Gasparin y Diéguez, 2018). Estos datos confirman que, a pesar del discurso modernizador, durante la gestión de Cambiemos se produjo una gran cantidad de nombramientos de funcionarios de forma irregular (Macco, 2020).

Respecto al empleo de personas con discapacidad encontramos un avance abrupto contra este colectivo. Entre las medidas que se han tomado están: supresión de las pensiones no contributivas; interrupción de entrega de las notebooks del programa Conectar Igualdad, que dotaba de computadoras personales a los estudiantes de escuelas de educación especial; incremento de servicios públicos, dificultando el acceso a "tarifas sociales"; e incremento del transporte público (Danel, 2019). En 2017, el Gobierno aprobó la Decisión Administrativa $N^{\circ} 12$, por la cual se congeló toda contratación de personal. Esta decisión no excluyó a las personas con discapacidad, en violación de la cuota laboral establecida en el marco normativo (Weicman, 2017). 
Esta multiplicidad de decisiones gubernamentales puso en riesgo los procesos de reproducción social de las personas en situación de discapacidad y la inscripción de sus necesidades en la agenda estatal. En el actual contexto, "en la era macrista" podemos identificar una matriz de intervención estatal que prioriza: la lógica de la protección focalizada por sobre la idea de seguridad social; la intensificación de los requerimientos para el acceso, lo que a todas luces produce barreras nuevas y profundizadas; los procesos de intervención profesional se complejizan en la medida que la red de seguridad social se hace laxa y desprotege; la complejidad de la intervención toma rostros de sufrimiento popular, desgaste profesional e incremento de la injusticia social (Gabrinetti y Danel, 2017, p. 7). En el siguiente cuadro, mostramos cómo fue -efectivamente- el cumplimiento del cupo laboral del $4 \%$ durante el período 2015-2019.

\begin{tabular}{l}
\hline Cuadro 3. Cupo laboral de personas con discapacidad entre 2015-2019 \\
\begin{tabular}{|l|c|c|c|c|}
\hline Año & Semestre & Total de trabajadores/as & Trabajadores/as con discapacidad & Cupo laboral (\%) \\
\hline 2015 & $2^{\circ}$ & 363.826 & 3.197 & 0,90 \\
\hline \multirow{2}{*}{2016} & $1^{\circ}$ & 319.122 & 3.008 & 0,94 \\
\cline { 2 - 5 } & $2^{\circ}$ & 339.369 & 3.081 & 0,91 \\
\hline \multirow{2}{*}{2017} & $1^{\circ}$ & 284.640 & 2.604 & 0,91 \\
\cline { 2 - 5 } & $2^{\circ}$ & 302.786 & 2.743 & 0,91 \\
\hline \multirow{2}{*}{2018} & $1^{\circ}$ & 314.828 & 2.740 & 0,87 \\
\hline 2019 & $2^{\circ}$ & 323.087 & 2.841 & 0,88 \\
\hline
\end{tabular}
\end{tabular}

Fuente: elaboración propia en base a los informes semestrales que elabora la Oficina Nacional de Empleo Público.

Como lo revela el cuadro 3, durante los primeros meses del gobierno de Mauricio Macri se produjo una reducción del empleo público del 12,3\%, como han precisado Blutman y Hoya (2019). Respecto al universo de trabajadoras y trabajadores con discapacidad empleados, se produjo una caída, en una proporción del $5,9 \%$. Posteriormente, a partir del segundo semestre de 2016, detectamos un incremento del empleo público, que vuelve a derrumbarse a principios de 2017. Finalmente, el mismo crece hasta el final del gobierno. Durante todo el gobierno, la cantidad de personas con discapacidad empleadas cae de 3197 a 2789 , lo que representa una proporción del 12,7\%. Ello permite explicar la detracción del cumplimiento del cupo laboral, que retrocedió al 0,85\%. Estos datos confirman que durante el gobierno de Cambiemos se produjo un retroceso en el cumplimiento de la cuota del $4 \%$. Veamos en el siguiente cuadro lo que ha sucedido con la obligación de reserva de las nuevas vacantes ${ }^{8}$.

8. Los datos provistos en los informes semestrales por parte de la Oficina Nacional de Empleo Público refieren a las altas y bajas totales de personal. Las bajas contemplan toda extinción de contrato, ya sea por despido, jubilación, renuncia u otras causas. Por su parte, las altas contemplan toda nueva relación contractual con nuevas personas, incluyendo todas las modalidades de contratación. 


\begin{tabular}{l}
\hline Cuadro 4. Evolución del empleo público entre semestres de 2015 y 2019 \\
\begin{tabular}{|l|c|c|c|c|}
\hline Semestres & $\begin{array}{c}\text { Variación de cantidad } \\
\text { de trabajadoras/es }\end{array}$ & $\begin{array}{c}\text { Variación de cantidad } \\
\text { de trabajadoras/es con } \\
\text { discapacidad }\end{array}$ & $\begin{array}{c}\text { Proporción personas } \\
\text { con discapacidad / } \\
\text { Variación }\end{array}$ & $\begin{array}{c}\text { Se cumplió la reserva } \\
\text { del 4\% }\end{array}$ \\
\hline $2^{\circ} 2015-1^{\circ} 2016$ & -44.704 & -189 & No corresponde & No corresponde \\
\hline $1^{\circ}-2^{\circ} 2016$ & 20.247 & 73 & $0,36 \%$ & No \\
\hline $2^{\circ} 2016-1^{\circ} 2017$ & -54.729 & -477 & No corresponde & No corresponde \\
\hline $1^{\circ}-2^{\circ} 2017$ & 18.146 & 139 & No corresponde & No corresponde \\
\hline $2^{\circ} 2017-1^{\circ} 2018$ & 12.042 & -3 & $1,22 \%$ & No \\
\hline $1^{\circ}-2^{\circ} 2018$ & 8.259 & 101 & No corresponde & No corresponde \\
\hline $2^{\circ} 2018-1^{\circ} 2019$ & 4.661 & -52 & &
\end{tabular} \\
\hline
\end{tabular}

Fuente: elaboración propia en base a los informes semestrales que elabora la Oficina Nacional de Empleo Público.

El cuadro 4 revela que hubo 5 períodos en los que aumentó el empleo (entre el primer y segundo semestre de 2016; y entre 2017 y los siguientes semestres). En ninguno de estos períodos se alcanzó la reserva de cargos del $4 \%$, e incluso hubo una reducción de trabajadoras y trabajadores con discapacidad cuando se incrementó la cantidad total de empleados (entre el segundo semestre de 2017 y el primer semestre de 2018; entre el segundo semestre de 2018 y el primer semestre de 2019). En síntesis, durante todo el período analizado, la política pública de cuotas no fue eficaz, es decir, no logró cumplir con los objetivos que se había planteado. En los años entre 2015-2019 no se alcanzó el cupo laboral del 4 \% ni se respetó la reserva de cargos ante nuevas contrataciones. Y, a diferencia de lo que ocurrió en el gobierno de Fernández de Kirchner, se produjo una reducción en la cantidad total de empleo público y en la contratación de personas con discapacidad.

\section{Reflexiones finales}

Los datos presentados en el texto confirman el incumplimiento de lo normado en el segundo párrafo del art. $8^{\circ}$ de la Ley $N^{\circ} 22.431$, vulnerando el derecho al empleo de las personas con discapacidad. En un informe presentado por el Centro de Estudios Legales y Sociales ante el Comité para la eliminación de todas las formas de discriminación racial de la Organización de Naciones Unidas se señala que los diversos argumentos esgrimidos para justificar este incumplimiento son múltiples y variados, pero ninguno explica cómo en un Estado de derecho se puede admitir el incumplimiento de la legislación vigente. Asimismo, se precisa que el ingreso a los empleos públicos responde a "cuestiones de clientelismo partidario o a contrataciones que no están sujetas a concursos o a métodos de selección que prioricen la inclusión de grupos vulnerables, sino todo lo contrario" (Centro de Estudios Legales y Sociales et al., 2004, p. 20).

Los procesos de producción de las políticas, en tanto forma de expresión de las capacidades estatales, evidencian que la inclusión laboral resulta un tema pendiente. La historia de inscripción en la agenda en 
torno a los sistemas de cuotas expresa un proceso de tramitación política que derivó en diseños y fundamentalmente de normas jurídicas.

En términos de eficacia, encontramos que el sistema de cuotas laborales en Argentina no ha sido satisfactorio. Registra casi 40 años sin alcanzar los objetivos que se propuso, lo que evidencia una acción de largo alcance, que se entrelaza a las formas en que social y culturalmente se valora el trabajo, los cuerpos de los sujetos y las expectativas de funcionamiento corporal. En el mismo sentido, alentamos a que -en futuros trabajos- se avance con evaluaciones profundas de las políticas públicas destinadas al colectivo con discapacidad donde pongan su atención en la eficiencia del sistema de cuotas. La evaluación de la eficiencia permitirá brindar elementos de juicio para identificar alternativas de acción que permitan obtener mejores resultados con igual cantidad de recursos o bien los mismos resultados con una mejor combinación o utilización de los recursos existentes (Nirenberg et al., 2007).

La necesidad de producir disputas de sentido con el capacitismo debiera ser promovida desde los responsables de las gestiones estatales y desde allí trabajar en el acompañamiento de trayectorias laborales en los múltiples ámbitos de la economía. Si el propio Estado incumple con los compromisos asumidos se limita la producción de exigibilidad al sector privado. El trabajo, como forma de valoración en la vida adulta, debe ser protegido e impulsado por el Estado.

Finalmente, destacamos que las políticas de acceso al empleo resultan una posibilidad de aminorar las marcas profundas de la desigualdad (Kessler, 2011; Neffa, 2012; Sconfienza, 2016), que se hacen presentes en las realidades de las personas en situación de discapacidad, desigualdades que asumen expresiones educativas y económicas. 
Acuña, C. y Bulit, L. (2010). Políticas sobre la discapacidad en la Argentina. El desafío de hacer realidad los derechos. Siglo veintiuno editores.

Alonso, A. y Cuenya, H. (2015). Los rengos de Perón. Crónica de un militante del Frente de Lisiados Peronistas. Colihue.

Álvarez, A. C. (2013). El impacto de los brotes de poliomielitis en las formas de organización ciudadana (Buenos Aires). Revista de Historia de la Medicina y Epistemología Medica, 1, pp. 1-17.

Arnau, S. (2013). La filosofía de vida independiente. Una estrategia política no violenta para una cultura de paz. Revista Latinoamericana de Educación Inclusiva, 7(1), pp. 93-112.

Banco Mundial (2018). Tasa de inactividad por edades (\% de la población en edad de trabajar) - Argentina. https:// datos.bancomundial.org/indicador/SP.POP.DPND?locations=AR.

Bertranou, J. (2013). Creación de agencias especializadas, capacidad estatal y coordinación interinstitucional. El caso de la Agencia Nacional de Seguridad Vial en Argentina. Revista Perspectivas de Políticas Públicas, 2(4), pp. 11-39. https://doi.org/10.18294/rppp.2013.621.

Bertranou, J. (2015). Capacidad estatal: revisión del concepto y algunos ejes de análisis y debate. Revista Estado y Políticas Públicas, (4), pp. 37-59. https://revistaeypp.flacso.org.ar/files/revistas/1433485077_dossier-2.pdf.

Blogna Tistuzza, S. H. (13-16 de octubre de 2020). Empleo público: la inclusión de las personas con discapacidad. Il Congreso Nacional de Estudios de Administración Pública. Asociación Argentina de Estudios de Administración Pública. https://aaeap.org.ar/wp-content/uploads/2020/11/BlognaSH.pdf.

Blutman, G. y Hoya, A. (2019). Reinventando la rueda-cuadrada: el empleo público en la modernización del Estado argentino (2015-2019). Revista Eletrônica de Administração, 25(2). https://doi.org/10.1590/14132311.244.90047.

Bourdieu, P. (1999). Meditaciones pascalianas. Anagrama.

Bregain, G. (2010). Etude sur le processus d'élaboration de la loi de protection intégrale des personnes handicapées en Argentine. Actes des Journées d'étude 'Droits de l'homme et recherche universitaire dans les Amériques".

Bregain, G. (2012). Historiar los derechos a la rehabilitación integral de las personas con discapacidad en Argentina (1946-1974). En L. Pantano, Discapacidad e Investigación: aportes desde la práctica (pp. 111-166). EDUCA.

Brogna, P. (2006). El nuevo paradigma de la discapacidad y el rol de los profesionales de la rehabilitación. Cad ESP, Ceará, 2(2), pp. 7-11. https://cadernos.esp.ce.gov.br/index.php/cadernos/article/view/21/19.

Bueno, C. P. y Andreu, A. (2009). Evaluación de las políticas de empleo para personas con discapacidad y formulación y coste económico de nuevas propuestas de integración laboral. CERMI.

Calfunao, C. et al. (2019). Luchas resemantizadas en contextos neoliberales: discapacidad mercantilizada. Entredichos, pp. 1-12. http://entredichos.trabajosocial.unlp.edu.ar/wp-content/uploads/sites/6/2019/12/EditadoCalfunao-Chavez-Danel-Martins-y-Oldani.pdf. 
Cannon, B. y Rangel, P (2020). Introducción: resurgimiento de la derecha en América Latina. Revista CIDOB d' Afers Internacionals, 126, pp. 07-16.

Caparrós, M. (1999). La Guerra Moderna: nuevas crónicas de larga distancia. Norma.

Castel, R. et al. (2013). Individuación, Precariedad, Inseguridad: ¿desinstitucionalización del presente? Paidós.

Centro de Estudios Legales y Sociales et al. (2004). Informe Alternativo presentado por el CELS ante el Comité para la eliminación de todas las formas de discriminación racial. CELS. http://www.cels.org.ar/common/documentos/cerd_personas_discapacidad.pdf.

Chudnovsky, M. (2017). La tensión entre mérito y confianza en la Alta Dirección Pública de América Latina. Revista del CLAD Reforma y Democracia, (69), pp. 5-40.

Club Marcelo J. Fitte (primer semestre de 1967). Revistas del Club Marcelo J. Fitte, Pro-superación del lisiado.

Comisión Nacional sobre la Desaparición de Personas (2010). Nunca Más. Eudeba.

Danel, P. M. (2019). Discapacidad y matriz colonial: el caso de las políticas de discapacidad en Argentina. En A. Yarza et al. (Coords.), Estudios críticos en discapacidad. Una polifonía desde América Latina (pp. 75-100). Consejo Latinoamericano de Ciencias Sociales.

Dussel, E. (2000). Europa, modernidad y eurocentrismo. En E. Lander (Comp.), La colonialidad del saber: eurocentrismo y ciencias sociales. Perspectivas latinoamericanas. CLACSO, Consejo Latinoamericano de Ciencias Sociales.

Ferrante, C. (2007). Algunas reflexiones sobre la situación de discapacidad en la Argentina en la actualidad. IV Jornadas de Jóvenes Investigadores. Instituto de Investigaciones Gino Germani. https://www.aacademica. org/000-024/173.

Ferrante, C. (2012). Luchas simbólicas en la definición del cuerpo discapacitado legitimo en el origen e institucionalización del campo del deporte adaptado de la Ciudad de Buenos Aires, Argentina (1950-1976). Revista Latinoamericana de Estudios sobre Cuerpos, Emociones y Sociedad, (9), pp. 38-51.

Ferrier, L. y Muller, V. (2008). Disabling Able. M/C Journal, 11(3). https://doi.org/10.5204/mcj.58.

Fuertes, G. y Ferreyra, M. (24 de abril de 2016). El ajuste laboral suma 1000 despidos por día desde que Macri llegó al poder. Tiempo Argentino. https://www.tiempoar.com.ar/nota/el-ajuste-laboral-suma-1000-despidospor-dia-desde-que-macri-llego-al-poder.

Fundación Par (2008). La discapacidad en Argentina: un diagnóstico de situación y políticas públicas vigentes a 2005. En C. Eroles y H. Fiamberti, Los derechos de las personas con discapacidad (Análisis de las convenciones internacionales y de la legislación vigente que los garantizan). Universidad de Buenos Aires.

Gabrinetti, M. A. y Danel, P. M. (2017). Intervenciones profesionales entre los sistemas de seguridad social y las protecciones. X Jornadas de Investigación, Docencia, Extensión y Ejercicio Profesional (X JIDEEP). Facultad de Trabajo Social de la Universidad Nacional de La Plata.

García, D. y Gradin, A. (2016). Neoliberalismo tardío y desestructuración del demos: el poder toma el poder. Revista de Estado y Políticas Públicas, 7(4), pp. 49-68.

Gasparin, J. y Diéguez, G. (2018). GPS del Estado: radiografía de 2017-2018. CIPPEC. https://www.cippec.org/ wp-content/uploads/2018/07/206-DPP-GP-GPS-GPS-del-Estado-radiograf\%C3\%ADa-de-2017-2018-Gasparin-J.-y-Di\%C3\%A9guez-G.-julio-de-2018.pdf. 
Guzmán, M. (2007). Evaluación de programas. Notas técnicas. CEPAL. https://repositorio.cepal.org/bitstream/ handle/11362/7321/S0700685_es.pdf?sequence=1\&isAllowed=y.

Honorable Cámara de Diputados de la Nación (1974). Diario de Sesiones de la Cámara de Diputados. Honorable Cámara de Diputados de la Nación.

Honorable Senado de la Nación (2016). Asamblea legislativa. Honorable Senado de la Nación. https://www.senado.gob.ar/parlamentario/sesiones/01-03-2016/00/downloadTac.

Honorable Senado de la Nación Argentina (1974). Diario de Sesiones de la Cámara de Senadores. Honorable Senado de la Nación Argentina.

Hudson, J. P. (2018). Gobiernos progresistas y cooperativismo: planes de empleo en la Argentina. Caderno CRH, 31(84). https://doi.org/10.1590/S0103-49792018000300012.

INDEC (2005). La población con discapacidad en la Argentina. Encuesta Nacional de Personas con Discapacidad. INDEC.

INDEC (2018a). Estudio Nacional sobre el Perfil de las Personas con Discapacidad. INDEC. https://www.indec. gob.ar/ftp/cuadros/poblacion/estudio_discapacidad_12_18.pdf.

INDEC (2018b). Mercado de trabajo. Tasas e indicadores socioeconómicos (EPH). INDEC. https://www.indec.gob. ar/uploads/informesdeprensa/mercado_trabajo_eph_4trim18.pdf.

International Disability Rights Monitor (2004). Monitoreo internacional de los derechos de las personas con discapacidad. Informe regional de las Américas. Centro para la Rehabilitación Internacional.

Joly, E. (2008). Por el derecho a ser explotados. Le Monde Diplomatique, 112, pp. 34-36. https://www.eldiplo. org/112-el-ocaso-de-washington/por-el-derecho-a-ser-explotados/.

Kessler, G. (2011). Exclusión social y desigualdad ¿nociones útiles para pensar la estructura social argentina? Lavboratorio. Revista de Estudios sobre Cambio Estructural y Desigualdad Social, (24), pp. 4-18.

Macco, G. D. (2020). Programa de Innovadores de Gobierno: los retos de la nueva gestión pública en Argentina. ICAP-Revista Centroamericana de Administración Pública, (78), pp. 238-249. https://doi.org/10.35485/ rcap78_13.

Mareño, M. (2015). Inclusión laboral de personas catalogadas como discapacitadas. Algunas reflexiones para un debate necesario. Trabajo y Sociedad, (25), pp. 405-442.

Nassif, C. P. y Nassif, R. E. (2019). La discapacidad desde un enfoque de derechos: a propósito de la legislación nacional argentina. Revista Latinoamericana en Discapacidad, Sociedad y Derechos Humanos, 3(2), pp. 58-74. http://redcdpd.net/revista/index.php/revista/article/view/162.

Neffa, J. C. (Coord.) (2012). De las políticas pasivas a las políticas activas: análisis comparativo de las políticas públicas de empleo (1989-2011). Empleo, desempleo \& políticas de empleo, (10). http://www.ceil-conicet.gov. ar/wp-content/uploads/2013/03/10polpasapolact.pdf.

Nirenberg, O. et al. (2007). Evaluar para la transformación. Paidós.

Ocampo, A. (2018). Las políticas de la mirada y la construcción epistemológica de la Educación Inclusiva: ¿en qué sentido la Educación Inclusiva es para todo el mundo? Polyphōnia. Revista de Educación Inclusiva. 2(1), pp. 15-51. 
O’Reilly, A. (2007). El derecho al trabajo decente de las personas con discapacidades. Oficina Internacional del Trabajo.

Organización de las Naciones Unidas (2006). Convención sobre los derechos de las personas con discapacidad. ONU. http://www.oas.org/DIL/esp/A-RES_61-106_spa.pdf.

Organización Iberoamericana de Seguridad Social (2014). Medidas para la promoción del empleo de personas con discapacidad en Iberoamérica. Organización Iberoamericana de Seguridad Social. http://repositoriocdpd. net:8080/bitstream/handle/123456789/1289/Inf_OISS_Estudiosobremedidaspromociondeempleo_2014. pdf?sequence $=1$.

Organización Internacional del Trabajo (12 de mayo de 2003). La OIT lanza el primer informe global sobre discriminación en el trabajo. Organización Internacional del Trabajo. https://www.ilo.org/global/about-the-ilo/newsroom/news/WCMS_071447/lang--es/index.htm.

Organización Internacional del Trabajo (2014). Lograr la igualdad de oportunidades en el empleo para las personas con discapacidades a través de la legislación. Directrices. Irish Aid. http://www.ilo.org/public/libdoc/ ilo/2014/114B09_183_span.pdf.

Organización Mundial de la Salud (2011). Informe mundial sobre la discapacidad. Organización Mundial de la Salud. http://www.who.int/disabilities/world_report/2011/es/.

Platero, R. (2012). Intersecciones: cuerpos y sexualidades en la encrucijada: temas contemporáneos. Bellaterra.

REDI et al. (2012). Informe Alternativo. situación de la discapacidad en Argentina 2008/2012. REDI. http://www. cels.org.ar/common/documentos/Informe\%20alternativo-\%201ra\%20evaluaci\%C3\%B3n\%20Argentina-\%20CRPD\%20-\%20FINAL.pdf.

Rengel, G. (2011). Frente de Lisiados Peronistas. Jornada Trabajo y discapacidad: estrategias de acción política. Defensoría del Pueblo de la Ciudad de Buenos Aires.

Rubio, F. (2009). Principios de normalización, integración e inclusión. Revista Digital Innovación y Experiencias Educativas, (19). http://educespecialjujuy.xara.hosting/index_htm_files/PRINCIPIO\%20DE\%20NORMALIZACION,\%20INTEGRACION\%20E\%2OINCLUSION.pdf.

Samaniego, P. (2006). Aproximación a la realidad de las personas con discapacidad en Latinoamérica. CERMI.

Sbriller, R. L. (2016). La revolución de los rengos. José Liborio Poblete Roa y el Frente de Lisiados Peronistas. Oficio, (2), pp. 93-115. http://revistaoficio.org/wp-content/uploads/2016/07/Oficio-N2-Completa.pdf.

Sconfienza, M. E. (2016). Demanda laboral y segmentos vulnerables. Empleo, desempleo y políticas de empleo, 25. http://www.ceil-conicet.gov.ar/wp-content/uploads/2016/08/EDPE25-Sconfienza.pdf.

Sheppard, C. (2011). Multiple Discrimination in the World of Work. Working Paper N 66. Oficina Internacional del Trabajo.

Skocpol, T. (1990). El Estado regresa al primer plano: estrategias de análisis en la investigación actual. En E. Torres-Rivas, Política. Educa.

Stang, M. F. (2011). Las personas con discapacidad en América Latina: del reconocimiento jurídico a la desigualdad real. CEPAL.

Trujillo, L. (2017). La Argentina kirchnerista: alcances y límites de una experiencia democrática sobre la distribución del ingreso (2003-2015). Polis, 46. https://journals.openedition.org/polis/12289. 
entre 2011 y 2019

Weicman, U. (Coord.) (2017). Evaluación sobre el estado de las personas con discapacidad en Argentina (20122017). Comité sobre los derechos de las personas con discapacidad. http://www.redi.org.ar/Documentos/ Informes/Informe-alternativo-Argentina-2017/Informe-Alternativo-Argentina.pdf. 